\title{
Branching Processes in a Lévy Random Environment
}

\author{
S. Palau ${ }^{1}$ J.C. Pardo ${ }^{1}$
}

Received: 28 June 2016 / Accepted: 6 August 2017 / Published online: 31 August 2017

(C) The Author(s) 2017. This article is published with open access at Springerlink.com

\begin{abstract}
In this paper, we introduce branching processes in a Lévy random environment. In order to define this class of processes, we study a particular class of non-negative stochastic differential equations driven by a white noise and Poisson random measures which are mutually independent. Following similar techniques as in Dawson and Li (Ann. Probab. 40:813-857, 2012) and Li and Pu (Electron. Commun. Probab. 17(33):1-13, 2012), we obtain existence and uniqueness of strong local solutions of such stochastic equations. We use the latter result to construct continuous state branching processes with immigration and competition in a Lévy random environment as a strong solution of a stochastic differential equation. We also study the long term behaviour of two interesting examples: the case with no immigration and no competition and the case with linear growth and logistic competition.
\end{abstract}

Keywords Continuous state branching processes in random environment - Stochastic differential equations $\cdot$ Strong solution $\cdot$ Immigration $\cdot$ Competition

Mathematics Subject Classification $60 \mathrm{G} 17 \cdot 60 \mathrm{G} 51 \cdot$ 60J80

\section{Introduction}

In many biological systems, when the population size is large enough, many birth and death events occur. Therefore, the dynamics of the population become difficult to describe. Under this scenario, continuous state models are good approximations of these systems and sometimes they can be simpler and computationally more tractable. Moreover, the qualitative behaviour of the approximate models may be easier to understand.

The simplest branching model in continuous time and space is perhaps the so called continuous state branching process (or CB-process for short). This model arises as the limit of

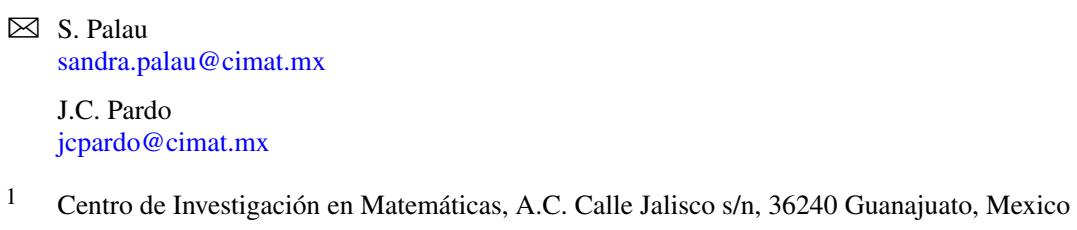


Galton-Watson processes; where individuals behave independently one from each other and each individual gives birth to a random number of offspring, with the same offspring distribution (see for instance Grimvall [19]). More precisely, a CB-process is a [0, $\infty]$-valued strong Markov process $Y=\left(Y_{t}, t \geq 0\right)$ with cádlág paths such that satisfies the branching property: for all $\theta \geq 0$ and $x, y \geq 0$,

$$
\mathbb{E}_{x+y}\left[e^{-\theta Y_{t}}\right]=\mathbb{E}_{x}\left[e^{-\theta Y_{t}}\right] \mathbb{E}_{y}\left[e^{-\theta Y_{t}}\right] .
$$

Moreover, its law is completely characterized by the latter identity, i.e.

$$
\mathbb{E}_{x}\left[e^{-\lambda Y_{t}}\right]=e^{-x u_{t}(\lambda)}, \quad t \geq 0,
$$

where $u$ is a differentiable function in $t$ satisfying

$$
\frac{\partial u_{t}(\lambda)}{\partial t}=-\psi\left(u_{t}(\lambda)\right), \quad u_{0}(\lambda)=\lambda,
$$

and $\psi$ satisfies

$$
\psi(\lambda)=-a \lambda+\gamma^{2} \lambda^{2}+\int_{(0, \infty)}\left(e^{-\lambda x}-1+\lambda x \mathbf{1}_{\{x<1\}}\right) \mu(\mathrm{d} x), \quad \lambda \geq 0,
$$

where $a, \gamma \in \mathbb{R}$ and $\mu$ is a measure concentrated on $(0, \infty)$ such that $\int_{(0, \infty)}\left(1 \wedge x^{2}\right) \mu(\mathrm{d} x)$ is finite. The function $\psi$ is known as the branching mechanism of $Y$. A process in this class can also be defined as the unique non-negative strong solution of the following stochastic differential equation (SDE for short)

$$
\begin{aligned}
Y_{t}= & Y_{0}+a \int_{0}^{t} Y_{s} \mathrm{~d} s+\int_{0}^{t} \sqrt{2 \gamma^{2} Y_{s}} \mathrm{~d} B_{s} \\
& +\int_{0}^{t} \int_{(0,1)} \int_{0}^{Y_{s-}} z \tilde{N}(\mathrm{~d} s, \mathrm{~d} z, \mathrm{~d} u)+\int_{0}^{t} \int_{[1, \infty)} \int_{0}^{Y_{s-}} z N(\mathrm{~d} s, \mathrm{~d} z, \mathrm{~d} u),
\end{aligned}
$$

where $B=\left(B_{t}, t \geq 0\right)$ is a standard Brownian motion, $N(\mathrm{~d} s, \mathrm{~d} z, \mathrm{~d} u)$ is a Poisson random measure independent of $B$, with intensity $\mathrm{d} s \mu(\mathrm{d} z) \mathrm{d} u$ and $\widetilde{N}$ is the compensated measure of $N$. Solutions for this type of SDE has been studied before, for instance: under a finite first moment condition, Bertoin and Le Gall [7] studied the weak existence and uniqueness of CB-processes without Feller diffusion component i.e. $\gamma=0$. Under the same condition, Dawson and Li [13] considered the strong existence and uniqueness. The most general case was studied by Caballero et al. [12].

Recently there has been a lot of interest in extending this model, in the sense that one would like to include immigration, competition or/and random environment. This interest comes from the fact that these new models arise as limits of discrete population models where there are interactions between individuals or where the offspring distribution depends on the environment (see for instance Lambert [26], Kawasu and Watanabe [22], Bansaye and Simatos [4] and the references therein).

Recall that a CB-process with immigration (or CBI-process) is a strong Markov process taking values in $[0, \infty]$, where 0 is no longer an absorbing state. It is characterized by a branching mechanism $\psi$ given by (2) and an immigration mechanism,

$$
\phi(u)=\mathrm{d} u+\int_{(0, \infty)}\left(1-e^{-u x}\right) v(\mathrm{~d} x), \quad u \geq 0,
$$


where $d \geq 0$ and $v$ is a measure supported in $(0, \infty)$ satisfying $\int_{0}^{\infty}(1 \wedge x) v(d x)<\infty$. It is well-known that if $\left(Y_{t}, t \geq 0\right)$ is a process in this class, then its semigroup is characterized by

$$
\mathbb{E}_{x}\left[e^{-\lambda Y_{t}}\right]=\exp \left\{-x u_{t}(\lambda)-\int_{0}^{t} \phi\left(u_{s}(\lambda)\right) \mathrm{d} s\right\}, \quad \text { for } \lambda \geq 0,
$$

where $u_{t}$ solves (1).

According to $\mathrm{Fu}$ and $\mathrm{Li}$ [18], under the condition that $\int_{(0, \infty)}\left(x \wedge x^{2}\right) \mu(\mathrm{d} x)$ is finite, a CBIprocess can be defined as the unique non-negative strong solution of the stochastic differential equation

$$
\begin{aligned}
Y_{t}= & Y_{0}+\int_{0}^{t}\left(\mathrm{~d}+a Y_{s}\right) \mathrm{d} s+\int_{0}^{t} \sqrt{2 \gamma^{2} Y_{s}} \mathrm{~d} B_{s} \\
& +\int_{0}^{t} \int_{(0, \infty)} \int_{0}^{Y_{s-}} z \tilde{N}(\mathrm{~d} s, \mathrm{~d} z, \mathrm{~d} u)+\int_{0}^{t} \int_{(0, \infty)} z M^{(i m)}(\mathrm{d} s, \mathrm{~d} z),
\end{aligned}
$$

where $M^{(i m)}(\mathrm{d} s, \mathrm{~d} z)$ is a Poisson random measures with intensity $\mathrm{d} s v(\mathrm{~d} z)$ independent of $B$ and $N$ (see also [13]).

CB processes with competition were first studied by Lambert [26], under the name of logistic branching processes, and more recently by Ma [30] and Beresticky et al. [6]. Under the assumption that

$$
\int_{(0, \infty)}\left(x \wedge x^{2}\right) \mu(\mathrm{d} x)<\infty
$$

the CB-process with competition is defined as the unique strong solution of the SDE

$$
Y_{t}=Y_{0}+a \int_{0}^{t} Y_{s} \mathrm{~d} s-\int_{0}^{t} \beta\left(Y_{s}\right) \mathrm{d} s+\int_{0}^{t} \sqrt{2 \gamma^{2} Y_{s}} \mathrm{~d} B_{s}+\int_{0}^{t} \int_{(0, \infty)} \int_{0}^{Y_{s-}} z \tilde{N}(\mathrm{~d} s, \mathrm{~d} z, \mathrm{~d} u)
$$

where $\beta$ is a continuous non-decreasing function on $[0, \infty)$ with $\beta(0)=0$, which is called the competition mechanism. The interpretation of the function $\beta$ is the following: in a given population of size $z$, an additional individual would be killed at rate $\beta(z)$. In his recent book, Pardoux [34] makes a systematic treatment of CB-processes with competition and continuous paths via stochastic differential equations. The author analyses the convergence of discrete state space models to continuous state space processes by the approach of SDEs driven by a white noise.

Branching processes in random environment (BPREs) were first introduced and studied in Smith and Wilkinson [37] and have attracted considerable interest in the last decade (e.g. $[1,10,27]$ and the references therein). BPREs are interesting since they are more realistic models compared with classical branching processes and, from the mathematical point of view, they have new properties such as another phase transition in the subcritical regime. Scaling limits in the finite variance case were conjectured by Keiding [24] who introduced Feller diffusions in random environment. This conjecture was proved by Kurtz [25] and more recently by Bansaye and Simatos [4] in more general cases.

There are new studies about the continuous state space setting, in all of them, the CBprocess in random environment is defined as a strong solution of a particular SDE. Böinghoff and Hutzenthaler [11] studied the case when the process possesses continuous paths. This 
process is the strong solution of the SDE

$$
Z_{t}=Z_{0}+a \int_{0}^{t} Z_{s} d s+\int_{0}^{t} \sqrt{2 \gamma^{2} Z_{s}} d B_{s}+\int_{0}^{t} Z_{s-} d S_{s},
$$

where the process $S=\left(S_{t}, t \geq 0\right)$ is a Brownian motion with drift which is independent of $B$. Bansaye and Tran [5] studied a cells dividing model which are infected by parasites. Informally, the quantity of parasites in a cell evolves as a Feller diffusion. The cells divide in continuous time at rate $r(x)$, which may depend on the quantity of parasites $x$ that they contain. When a cell divides, a random fraction $\theta$ of parasites goes in the first daughter cell and the rest in the second one. In each division, they only keep one cell and consider the quantity of parasites inside. Assuming that the rate $r$ is constant and $\theta$ is a r.v. in $(0,1)$ with distribution $F$, the model follows a Feller diffusion with multiplicative jumps of independent sizes distributed as $F$ and which occurs at rate $r$. In particular, the model can be described as in (4) with $S$ satisfying

$$
S_{t}=r \int_{0}^{t} \int_{(0,1)}(\theta-1) M(\mathrm{~d} s, \mathrm{~d} \theta)
$$

where $M$ is a Poisson random measure with intensity $\mathrm{d} s F(\mathrm{~d} \theta)$. Inspired in this model, Bansaye et al. [3] studied more general CB-processes in random environment which are driven by Lévy processes whose paths are of bounded variation and the branching mechanism possesses a first moment condition. They were called CB-processes with catastrophes motivated by the fact that the presence of a negative jump in the random environment represents that a proportion of the population, following the dynamics of the CB-process, is killed. The process is defined as the unique non negative strong solution of the SDE

$$
Z_{t}=Z_{0}+a \int_{0}^{t} Z_{s} \mathrm{~d} s+\int_{0}^{t} \sqrt{2 \gamma^{2} Z_{s}} \mathrm{~d} B_{s}+\int_{0}^{t} \int_{(0, \infty)} \int_{0}^{Z_{s-}} z \tilde{N}(\mathrm{~d} s, \mathrm{~d} z, \mathrm{~d} u)+\int_{0}^{t} Z_{s-} \mathrm{d} S_{s},
$$

where

$$
S_{t}=\int_{0}^{t} \int_{(0, \infty)}(m-1) M(\mathrm{~d} s, \mathrm{~d} m)
$$

$M$ is a Poisson random measure independent of $N$ and $B$, with intensity $\mathrm{d} s v(\mathrm{~d} m)$ and $v$ is a measure concentrated on $(0, \infty)$ such that

$$
0<\int_{(0, \infty)}(1 \wedge|m-1|) v(\mathrm{~d} m)<\infty
$$

Palau and Pardo [32] considered a CB-process with immigration and general branching mechanism in a Brownian random environment. In other words, the authors in [32] consider the following SDE,

$$
\begin{aligned}
Z_{t}= & Z_{0}+\int_{0}^{t}\left(\mathrm{~d}+a Z_{s}\right) \mathrm{d} s+\int_{0}^{t} \sqrt{2 \gamma^{2} Y_{s}} \mathrm{~d} B_{s}+\int_{0}^{t} Z_{s} \mathrm{~d} S_{s} \\
& +\int_{0}^{t} \int_{(0, \infty)} \int_{0}^{Z_{s-}} z \tilde{N}(\mathrm{~d} s, \mathrm{~d} z, \mathrm{~d} u)+\int_{0}^{t} \int_{(0, \infty)} z M^{(i m)}(\mathrm{d} s, \mathrm{~d} z),
\end{aligned}
$$


where $S$ is a Brownian motion with drift, $\mathrm{d}$ is a non-negative number, $M^{(i m)}(\mathrm{d} s, \mathrm{~d} z)$ is a Poisson random measure with intensity $\mathrm{d} s v(\mathrm{~d} z)$ satisfying

$$
\int_{(0, \infty)}(1 \wedge z) v(d z)<\infty
$$

and the processes $S, B, N$ and $M_{1}$ are mutually independent. In all these manuscripts, the authors proved the existence of such processes and obtained the speed of extinction. As in the case of BPREs, there is a phase transition in the subcritical regime.

Evans et al. [17] consider a population living in a spatially heterogeneous environment with $n$ different patches. These patches may represent distinct habitats, patches of the same habitat type or combinations thereof. The population in the $i$-th patch at time $t \geq 0$ is given by

$$
X_{t}^{(i)}=X_{0}^{(i)}+\int_{0}^{t} X_{s}^{(i)}\left(\mu_{i}-k_{i} X_{s}^{(i)}\right) \mathrm{d} s+\int_{0}^{t} X_{s}^{(i)} \mathrm{d} E_{s}^{(i)},
$$

where $\mu_{i}$ is the rate of growth in the patch $i, k_{i}$ represents the competition in the patch $i$, and $E_{t}^{(i)}=\sum_{j} \gamma_{i j} B_{t}^{(j)}$, with $\left(B_{t}^{(1)}, \ldots, B_{t}^{(n)}\right)$ a standard Brownian motion on $\mathbb{R}^{n}$. They assume that the fraction of population in patch $i$ is equal to $\alpha_{i}$ all the time. Therefore if $\alpha_{1}, \ldots, \alpha_{n} \geq 0$ are such that $\sum_{i \leq n} \alpha_{i}=1$, we have $X_{t}^{(i)}=\alpha_{i} X_{t}$ where $X_{t}=\sum_{i \leq n} X_{t}^{(i)}$. In this case, the process $X$ satisfies the SDE

$$
X_{t}=X_{0}+\int_{0}^{t} X_{s} \sum_{i=1}^{n} \alpha_{i}\left(\mu_{i}-k_{i} \alpha_{i} X_{s}\right) \mathrm{d} s+\int_{0}^{t} X_{s} \sum_{i=1}^{n} \alpha_{i} \mathrm{~d} E_{s}^{(i)}
$$

In this paper, one of our aims is to construct a continuous state branching processes with immigration and competition in a Lévy random environment as a strong solution of a SDE. In order to do so, we study a particular class of non-negative SDEs driven by a white noise and Poisson random measures which are mutually independent. The existence and uniqueness of strong solutions are established under some general conditions that allows us to consider the case when the strong solution explodes at a finite time. This result is of particular interest on its own.

The remainder of the paper is structured as follows. In Sect. 2, we study strong solutions of SDEs which are driven by a white noise and Poisson random measures which are mutually independent. Section 3 is devoted to the construction of CBI-processes with competition in a Lévy random environment which is an extension of the models introduced in Bansaye et al. [3] and Palau and Pardo [32]. In particular, we study the long term behaviour of CBprocesses in a Lévy random environment. We finish our exposition by studying a population model with competition in a Lévy random environment which can be considered as an extension of the model of Evans et al. [17]. In particular, we study its long time behaviour and the Laplace transform of its first passage time below a level under the assumption that the environment has no negative jumps.

\section{Stochastic Differential Equations}

Stochastic differential equations with jumps have been playing an ever more important role in various domains of applied probability theory such as financial mathematics or mathematical biology. Under Lipschitz conditions, the existence and uniqueness of strong solutions of SDEs with jumps can be established by arguments based on Gronwall's inequality and 
results on continuous-type equation, see for instance the monograph of Ikeda and Watanabe [21]. In view of the results of Fu and $\mathrm{Li} \mathrm{[18]} \mathrm{and} \mathrm{Dawson} \mathrm{and} \mathrm{Li} \mathrm{[14]} \mathrm{weaker} \mathrm{conditions}$ would be sufficient for the existence and uniqueness of strong solutions for one-dimensional equations.

Motivated by describing CBI processes via SDEs, Fu and Li [18] studied general stochastic equations that describes non-negative processes with jumps under general conditions. More precisely, the authors in [18] provided criteria for the existence and uniqueness of strong solutions of SDEs which are driven by a Brownian motion and Poisson random measures. Dawson and Li [14] studied similar SDEs but instead of considering a Brownian motion in the diffusion term, they replaced it by a white noise. The main idea of their criteria is to assume monotonicity conditions on the kernel which is associated with the compensated Poisson integral term so that the continuity condition can be weakened. In this setting, $\mathrm{Li}$ and $\mathrm{Pu}$ [29], weakened the monotonicity assumption but only in the case when the white noise is replaced by a Brownian motion. Nonetheless, their criteria do not include the case where the branching mechanism of a CBI process has infinite mean. This excludes some interesting models that can be of particular interest for applications.

Our goal in this section is to describe a general one-dimensional SDE driven by a white noise and Poisson random measures that may relax the moment condition of the previous works. The main idea is first to combine the works of Dawson and $\mathrm{Li}$ [14] and $\mathrm{Li}$ and $\mathrm{Pu}$ [29] and later on weaken the moment condition. This may imply that the process has the possibility of explosion at finite time. This will allow us to define branching processes in more general random environment that those considered by Bansaye et al. [3] and Palau and Pardo [32].

Let $E, U$ and $V$ be separable topological spaces whose topologies can be defined by complete metrics. Suppose that $\pi, \mu$ and $v$ are $\sigma$-finite Borel measures defined on $E, U$ and $V$, respectively. We say that the parameters $(b, \sigma, g, h)$ are admissible if

(i) $b: \mathbb{R}_{+} \rightarrow \mathbb{R}$ is a continuous function such that $b(0) \geq 0$,

(ii) $\sigma: \mathbb{R}_{+} \times E \rightarrow \mathbb{R}_{+}$is a Borel function, continuous in the first entry such that $\sigma(0, x)=0$, for all $x \in E$,

(iii) $g: \mathbb{R}_{+} \times U \rightarrow \mathbb{R}$ is a Borel functions such that $g(x, u)+x \geq 0$ for $x \geq 0$ and $u \in U$,

(iv) $h: \mathbb{R}_{+} \times V \rightarrow \mathbb{R}$ is a Borel function such that $h(0, v)=0$ and $h(x, v)+x \geq 0$ for $x>0$ and $v \in V$.

Let $W$ be a white noise on $(0, \infty) \times E$ with intensity $\mathrm{d} s \pi(\mathrm{d} z)$. Let $M$ and $N$ be two Poisson random measures defined on $\mathbb{R}_{+} \times U$ and $\mathbb{R}_{+} \times V$, respectively, with intensities given by $\mathrm{d} s \mu(\mathrm{d} u)$ and $\mathrm{d} s v(\mathrm{~d} v)$. We also suppose that $B, M$ and $N$ are mutually independent. The compensated measure of $N$ is denoted by $\widetilde{N}$.

Let $\widetilde{U}$ be a subset in $U$ such that $\mu(U \backslash \widetilde{U})<\infty$. For our purposes, we consider the following conditions on the parameters $(b, \sigma, g, h)$ :

(a) For each $n$, there is a positive constant $A_{n}$ such that

$$
\int_{\widetilde{U}}(|g(x, u)| \wedge 1) \mu(\mathrm{d} u) \leq A_{n}(1+x), \quad \text { for every } x \in[0, n] .
$$

(b) Let $b(x)=b_{1}(x)-b_{2}(x)$, where $b_{1}$ is a continuous function and $b_{2}$ is a non-decreasing continuous function. For each $n \geq 0$, there is a non-decreasing concave function $z \mapsto$ $r_{n}(z)$ on $\mathbb{R}_{+}$satisfying $\int_{0+} r_{n}(z)^{-1} \mathrm{~d} z=\infty$ and

$$
\left|b_{1}(x)-b_{1}(y)\right|+\int_{\widetilde{U}}|g(x, u) \wedge n-g(y, u) \wedge n| \mu(\mathrm{d} u) \leq r_{n}(|x-y|)
$$

for every $0 \leq x, y \leq n$. 
(c) For each $n \geq 0$ and $v \in V$, the function $x \mapsto x+h(x, v)$ is non-decreasing and there is a positive constant $B_{n}$ such that for every $0 \leq x, y \leq n$,

$$
\int_{E}|\sigma(x, u)-\sigma(y, u)|^{2} \pi(\mathrm{d} u)+\int_{V}\left(|l(x, y, v)| \wedge(l(x, y, v))^{2}\right) v(\mathrm{~d} v) \leq B_{n}|x-y|
$$

where $l(x, y, v)=h(x, v)-h(y, v)$.

A $[0, \infty]$-valued process $Z=\left(Z_{t}, t \geq 0\right)$ with càdlàg paths is called a solution of

$$
\begin{aligned}
Z_{t}= & Z_{0}+\int_{0}^{t} b\left(Z_{s}\right) \mathrm{d} s+\int_{0}^{t} \int_{E} \sigma\left(Z_{s}, u\right) \mathrm{d} W(\mathrm{~d} s, \mathrm{~d} u) \\
& +\int_{0}^{t} \int_{U} g\left(Z_{s-}, u\right) M(\mathrm{~d} s, \mathrm{~d} u)+\int_{0}^{t} \int_{V} h\left(Z_{s-}, v\right) \tilde{N}(\mathrm{~d} s, \mathrm{~d} v),
\end{aligned}
$$

if it satisfies (5) up to the time $\tau_{n}:=\inf \left\{t \geq 0: Z_{t} \geq n\right\}$ for all $n \geq 1$, and $Z_{t}=\infty$ for all $t \geq \tau:=\lim _{n \rightarrow \infty} \tau_{n}$. We say that $Z$ is a strong solution if, in addition, it is adapted to the augmented natural filtration generated by $W, M$ and $N$. (See for instance Definition IV.2.1 of Ikeda and Watanabe [21] for further details of solutions that admits explosions.)

Proposition 1 Suppose that $(b, \sigma, g, h)$ are admissible parameters satisfying conditions (a), (b) and (c). Then, the stochastic differential equation (5) has a unique non-negative strong solution. The process $Z=\left(Z_{t}, t \geq 0\right)$ is a Markov process and its infinitesimal generator $\mathcal{L}$ satisfies, for every $f \in C_{b}^{2}\left(\overline{\mathbb{R}}_{+}\right),{ }^{1}$

$$
\begin{aligned}
\mathcal{L} f(x)= & b(x) f^{\prime}(x)+\frac{1}{2} f^{\prime \prime}(x) \int_{E} \sigma^{2}(x, u) \pi(\mathrm{d} u)+\int_{U}(f(x+g(x, u))-f(x)) \mu(\mathrm{d} u) \\
& +\int_{V}\left(f(x+h(x, v))-f(x)-f^{\prime}(x) h(x, v)\right) v(\mathrm{~d} v)
\end{aligned}
$$

Proof Observe that Theorem 6.1 in $\mathrm{Li}$ and $\mathrm{Pu}$ [29] still holds if we replace the Brownian motion integral term by a white noise, i.e. in the setting of Dawson and Li [14], the result is true if we consider a white noise $W$ in $(0, \infty) \times E$ and a function $\sigma(x, u)$ on $\mathbb{R}_{+} \times E$ such that (c) is satisfied. That is to say, following the steps of $\mathrm{Li}$ and $\mathrm{Pu}$ [29], we need to replace $\sigma(x)$ by $\int_{E} \sigma(x, u) \pi(\mathrm{d} u)$ and $|\sigma(x)-\sigma(y)|^{2}$ by $\int_{E}|\sigma(x, u)-\sigma(y, u)|^{2} \pi(\mathrm{d} u)$.

Then, we can apply the theorem to the functions

$$
\begin{aligned}
b_{n}(x) & =b(x \wedge n), & & \sigma_{n}(x, u)=\sigma(x \wedge n, u) \\
g_{0}^{n}(x, u) & =h(x \wedge n, u) \wedge n & & g_{1}^{n}(x, u)=g(x \wedge n, u) \wedge n
\end{aligned}
$$

In other words, we deduce that for $n>0$ there is a unique non-negative strong solution of

$$
\begin{aligned}
Z_{t}^{(n)}= & Z_{0}+\int_{0}^{t} b\left(Z_{s}^{(n)} \wedge n\right) \mathrm{d} s+\int_{0}^{t} \int_{E} \sigma\left(Z_{s}^{(n)} \wedge n, u\right) W(\mathrm{~d} s, \mathrm{~d} u) \\
& +\int_{0}^{t} \int_{U}\left(g\left(Z_{s-}^{(n)} \wedge n, u\right) \wedge n\right) M(\mathrm{~d} s, \mathrm{~d} u)+\int_{0}^{t} \int_{V}\left(h\left(Z_{s-}^{(n)} \wedge n, v\right) \wedge n\right) \tilde{N}(\mathrm{~d} s, \mathrm{~d} v) .
\end{aligned}
$$

${ }^{1} \mathbb{R}_{+}=[0, \infty), \overline{\mathbb{R}}_{+}=[0, \infty]$ and $C_{b}^{2}\left(\overline{\mathbb{R}}_{+}\right)=\{$twice differentiable functions such that $f(\infty)=0\}$ 
Now, we proceed to show that there is a unique non-negative strong solution to the SDE (5). In order to do so, we first define $\tau_{m}=\inf \left\{t \geq 0: Z_{t}^{(m)} \geq m\right\}$, for $m \geq 0$, and then we prove that the sequence $\left(\tau_{m}, m \geq 0\right)$ is non-decreasing and that $Z_{t}^{(m)}=Z_{t}^{(n)}$ for $m \leq n$ and $t<\tau_{m}$.

Since the Poisson random measures are independent, they do not jump simultaneously. Therefore, by using that $Z_{t}^{(m)} \in[0, m]$ for $t \in\left[0, \tau_{m}\right)$, we obtain that for $0 \leq t<\tau_{m}$,

$$
g\left(Z_{t}^{(m)}, u\right) \leq m \quad \text { and } \quad h\left(Z_{t}^{(m)}, v\right) \leq m, \quad u \in U, v \in V .
$$

This implies that $Z_{t}^{(m)}$ satisfies (5) on the interval $\left[0, \tau_{m}\right)$. For $m \leq n$, let $\left(Y_{t}^{(n)}, t \geq 0\right)$ be the strong solution to

$$
\begin{aligned}
Y_{t}^{(n)}= & Z_{\tau_{m}-}^{(m)}+\int_{0}^{t} b\left(Y_{s}^{(n)} \wedge n\right) \mathrm{d} s+\int_{0}^{t} \int_{E} \sigma\left(Y_{s}^{(n)} \wedge n, u\right) W\left(\mathrm{~d}\left(\tau_{m}+s\right), \mathrm{d} u\right) \\
& +\int_{0}^{t} \int_{U}\left(g\left(Y_{s-}^{(n)} \wedge n, u\right) \wedge n\right) M\left(\tau_{m}+\mathrm{d} s, \mathrm{~d} u\right) \\
& +\int_{0}^{t} \int_{V}\left(h\left(Y_{s-}^{(n)} \wedge n, v\right) \wedge n\right) \tilde{N}\left(\tau_{m}+\mathrm{d} s, \mathrm{~d} v\right)
\end{aligned}
$$

We define $\tilde{Y}_{t}^{(n)}=Z_{t}^{(m)}$ for $0 \leq t<\tau_{m}$ and $\tilde{Y}_{t}^{(n)}=Y_{t-\tau_{m}}^{(n)}$ for $t \geq \tau_{m}$. Note that $\left(\tilde{Y}_{t}^{(n)}, t \geq 0\right)$ is solution to (7). From the strong uniqueness, we deduce that $Z_{t}^{(n)}=\widetilde{Y}_{t}^{(n)}$ for all $t \geq 0$. In particular, we have that $Z_{t}^{(n)}=Z_{t}^{(m)}<m$ for $0 \leq t<\tau_{m}$. Consequently, the sequence $\left(\tau_{m}, m \geq 0\right)$ is non-decreasing.

Next, we define the process $Z=\left(Z_{t}, t \geq 0\right)$ as

$$
Z_{t}= \begin{cases}Z_{t}^{(m)} & \text { if } t<\tau_{m}, \\ \infty & \text { if } t \geq \lim _{m \rightarrow \infty} \tau_{m} .\end{cases}
$$

It is not difficult to see that $Z$ is a weak solution to (5). Finally, we consider two solutions to (5), $Z^{\prime}$ and $Z^{\prime \prime}$, and consider $\tau_{m}^{\prime}=\inf \left\{t \geq 0: Z_{t}^{\prime} \geq m\right\}, \tau_{m}^{\prime \prime}=\inf \left\{t \geq 0: Z_{t}^{\prime \prime} \geq m\right\}$ and $\tau_{m}=\tau_{m}^{\prime} \wedge \tau_{m}^{\prime \prime}$. Therefore $Z^{\prime}$ and $Z^{\prime \prime}$ satisfy (7) on $\left[0, \tau_{m}\right)$, implying that they are indistinguishable on $\left[0, \tau_{m}\right)$. Observe that $g$ and $h$ are finite functions. Then, the processes $Z^{\prime}$ and $Z^{\prime \prime}$ cannot have a jump of infinite size. This implies that if $\tau_{\infty}=\lim _{m \rightarrow \infty} \tau_{m}<\infty$, then $Z_{\tau_{\infty-}}^{\prime}=Z_{\tau_{\infty-}}^{\prime \prime}=\infty$. Since after this time both processes are equal to $\infty$, we obtain that $Z^{\prime}$ and $Z^{\prime \prime}$ are indistinguishable. In other words, there is a unique strong solution to (5). The strong Markov property follows from the fact that we have a strong solution, the integrators are Lévy processes and the integrand functions doesn't depend on time. (See Theorem V.32 in Protter [35], where the Lipschitz continuity is just to guarantee the existence and uniqueness of the solution.) Finally, from Itô's formula it is easy to show that the infinitesimal generator of $Z$ is given by (6).

It is important to note that the SDEs (5) can be driven by a finite number of Poisson random measures which are mutually independent. By rewriting the measures, we could apply the previous theorem.

\section{CBI-Processes with Competition in a Lévy Random Environment}

In this section, we construct a branching model in continuous time and space that is affected by a random environment as the unique strong solution of a SDE that satisfies the conditions of Theorem 1. In this model, the random environment is driven by a general Lévy process. 
Our model is a natural extension of the CB-processes in random environment studied by Bansaye et al. [3], in the case where the Lévy process has paths of bounded variation, and by Palau and Pardo [32], in the case where the random environment is driven by a Brownian motion with drift.

In order to define CBI-processes in a Lévy random environment (CBILRE for short), we first introduce the objects that are involve on the branching, immigration and environmental parts. Recall that the associated branching mechanism $\psi$, satisfies

$$
\psi(\lambda)=-a \lambda+\gamma^{2} \lambda^{2}+\int_{(0, \infty)}\left(e^{-\lambda x}-1+\lambda x \mathbf{1}_{\{x<1\}}\right) \mu(\mathrm{d} x), \quad \lambda \geq 0,
$$

and the immigration mechanism is given by

$$
\phi(u)=\mathrm{d} u+\int_{(0, \infty)}\left(1-e^{-u t}\right) v(\mathrm{~d} t), \quad u \geq 0,
$$

where $a, \gamma \in \mathbb{R}, \mathrm{d} \geq 0$ and $\nu$ and $\mu$ are measures supported in $(0, \infty)$ satisfying

$$
\int_{(0, \infty)}(1 \wedge x) v(\mathrm{~d} x)+\int_{(0, \infty)}\left(1 \wedge x^{2}\right) \mu(\mathrm{d} x)<\infty .
$$

The competition mechanism is given by a continuous non-decreasing function $\beta$ on $[0, \infty)$ with $\beta(0)=0$. For the branching part, we introduce $B^{(b)}=\left(B_{t}^{(b)}, t \geq 0\right)$ a standard Brownian motion and $N^{(b)}(\mathrm{d} s, \mathrm{~d} z, \mathrm{~d} u)$ a Poisson random measure with intensity $\mathrm{d} s \mu(\mathrm{d} z) \mathrm{d} u$. We denote by $\widetilde{N}^{(b)}$ the compensated measure of $N^{(b)}$. The immigration term is given by a Poisson random measure $M^{(i m)}(\mathrm{d} s, \mathrm{~d} z)$ with intensity $\mathrm{d} s v(\mathrm{~d} z)$. Finally, for the environmental term, we introduce $B^{(e)}=\left(B_{t}^{(e)}, t \geq 0\right)$ a standard Brownian motion and $N^{(e)}(\mathrm{d} s, \mathrm{~d} z)$ a Poisson random measure in $\mathbb{R}_{+} \times \mathbb{R}$ with intensity $\mathrm{d} s \pi(\mathrm{d} z)$, where $\pi$ is a measure concentrated on $\mathbb{R} \backslash\{0\}$ such that

$$
\int_{\mathbb{R}}\left(1 \wedge z^{2}\right) \pi(\mathrm{d} z)<\infty
$$

and $\tilde{N}^{(e)}$ its compensated version. We will assume that all the objects involve are mutually independent.

A CB-process in a Lévy random environment with immigration and competition is defined as the solution of the SDE

$$
\begin{aligned}
Z_{t}= & Z_{0}+\int_{0}^{t}\left(\mathbf{d}+a Z_{s}\right) \mathrm{d} s+\int_{0}^{t} \sqrt{2 \gamma^{2} Z_{s}} \mathrm{~d} B_{s}^{(b)} \\
& -\int_{0}^{t} \beta\left(Z_{s}\right) \mathrm{d} s+\int_{0}^{t} \int_{(0, \infty)} z M^{(i m)}(\mathrm{d} s, \mathrm{~d} z)+\int_{0}^{t} Z_{s-} \mathrm{d} S_{s} \\
& +\int_{0}^{t} \int_{(0,1)} \int_{0}^{Z_{s-}} z \widetilde{N}^{(b)}(\mathrm{d} s, \mathrm{~d} z, \mathrm{~d} u)+\int_{0}^{t} \int_{[1, \infty)} \int_{0}^{Z_{s-}} z N^{(b)}(\mathrm{d} s, \mathrm{~d} z, \mathrm{~d} u),
\end{aligned}
$$

where

$$
S_{t}=\alpha t+\sigma B_{t}^{(e)}+\int_{0}^{t} \int_{(-1,1)}\left(e^{z}-1\right) \tilde{N}^{(e)}(\mathrm{d} s, \mathrm{~d} z)+\int_{0}^{t} \int_{\mathbb{R} \backslash(-1,1)}\left(e^{z}-1\right) N^{(e)}(\mathrm{d} s, \mathrm{~d} z),
$$

with $\alpha \in \mathbb{R}$ and $\sigma \geq 0$. 
Theorem 1 The stochastic differential equation (8) has a unique non-negative strong solution. The process $Z=\left(Z_{t}, t \geq 0\right)$ has the Markov property and its infinitesimal generator $\mathcal{A}$ satisfies, for every $f \in C_{b}^{2}\left(\overline{\mathbb{R}}_{+}\right)$, and $x \in \mathbb{R}_{+}$

$$
\begin{aligned}
\mathcal{A} f(x)= & (a x+\alpha x-\beta(x)+\mathbf{d}) f^{\prime}(x)+\int_{(0, \infty)}(f(x+z)-f(x)) v(\mathrm{~d} z) \\
& +\left(\gamma^{2} x+\frac{\sigma^{2}}{2} x^{2}\right) f^{\prime \prime}(x)+x \int_{(0, \infty)}\left(f(x+z)-f(x)-z f^{\prime}(x) \mathbf{1}_{\{z<1\}}\right) \mu(\mathrm{d} z) \\
& +\int_{\mathbb{R}}\left(f\left(x e^{z}\right)-f(x)-x\left(e^{z}-1\right) f^{\prime}(x) \mathbf{1}_{\{|z|<1\}}\right) \pi(\mathrm{d} z) .
\end{aligned}
$$

Proof The proof of this result is a straightforward application of Proposition 1. We take $E=\{1,2\}$,

$$
\begin{aligned}
& U=\{1\} \times[1, \infty) \times \mathbb{R}_{+} \cup\{2\} \times \mathbb{R} \backslash(-1,1) \times\{0\} \cup\{3\} \times \mathbb{R}_{+} \times\{0\}, \\
& \widetilde{U}=\{1\} \times[1, \infty) \times \mathbb{R}_{+} \cup\{2\} \times(-\infty,-1) \times\{0\} \cup\{3\} \times \mathbb{R}_{+} \times\{0\}, \\
& V=\{1\} \times(0,1) \times \mathbb{R}_{+} \cup\{2\} \times(-1,1) \times\{0\},
\end{aligned}
$$

with associated Poisson random measures

$$
\begin{aligned}
M(\mathrm{~d} r, \mathrm{~d} s, \mathrm{~d} z, \mathrm{~d} u):= & \delta_{1}(\mathrm{~d} r) N^{(b)}(\mathrm{d} s, \mathrm{~d} z, \mathrm{~d} u)+\delta_{2}(\mathrm{~d} r) N^{(e)}(\mathrm{d} s, \mathrm{~d} z) \delta_{0}(\mathrm{~d} u) \\
& +\delta_{3}(\mathrm{~d} r) M^{(i m)}(\mathrm{d} s, \mathrm{~d} z) \delta_{0}(\mathrm{~d} u) \\
N(\mathrm{~d} r, \mathrm{~d} s, \mathrm{~d} z, \mathrm{~d} u):= & \delta_{1}(\mathrm{~d} r) N^{(b)}(\mathrm{d} s, \mathrm{~d} z, \mathrm{~d} u)+\delta_{2}(\mathrm{~d} r) N^{(e)}(\mathrm{d} s, \mathrm{~d} z) \delta_{0}(\mathrm{~d} u)
\end{aligned}
$$

respectively; and white noise in $\mathbb{R} \times E$ given by

$$
W(\mathrm{~d} s, \mathrm{~d} u)=B^{(b)}(\mathrm{d} s) \delta_{1}(\mathrm{~d} u)+B^{(e)}(\mathrm{d} s) \delta_{2}(\mathrm{~d} u),
$$

with intensity $\mathrm{d} s\left(\delta_{1}(\mathrm{~d} u)+\delta_{2}(\mathrm{~d} u)\right)$, where $\delta$ denotes the Dirac measure. We also take the functions $b(x)=a x-\beta(x)+\mathbf{d}, \sigma(x, u)=\sqrt{2 \gamma^{2} x} \mathbf{1}_{\{u=1\}}+\sigma x \mathbf{1}_{\{u=2\}}$,

$$
\begin{aligned}
& g(x, r, z, u)=\mathbf{1}_{\{r=1\}} z \mathbf{1}_{\{u<x\}}+\mathbf{1}_{\{r=2\}} x\left(e^{z}-1\right) \mathbf{1}_{\{u=0\}}+\mathbf{1}_{\{r=3\}} z \mathbf{1}_{\{u=0\}}, \\
& h(x, r, z, u)=\mathbf{1}_{\{r=1\}} z \mathbf{1}_{\{u<x\}}+\mathbf{1}_{\{r=2\}} x\left(e^{z}-1\right) \mathbf{1}_{\{u=0\}},
\end{aligned}
$$

which are admissible and verify conditions (a), (b) and (c). The result then follows by applying Proposition 1.

While this paper was under review, we were aware that CBILRE-processes under a first moment condition were introduced by $\mathrm{He}$ et al. [20]. The authors provide a direct construction of their model and gave sufficient and necessary conditions for the process to be absorbed at 0 .

Similarly to the cases of Bansaye et al. [3] and Palau and Pardo [32], we can compute the Laplace transform of a reweighted version of $Z$ given the environment and under the assumption that $\beta \equiv 0$. In order to do so, we define the following hypothesis

$$
\int_{[1, \infty)} x \mu(\mathrm{d} x)<\infty .
$$


Observe that from our assumption, $\left|\psi^{\prime}(0+)\right|<\infty$ and

$$
\psi^{\prime}(0+)=-a-\int_{[1, \infty)} x \mu(\mathrm{d} x)
$$

For the sequel, we define the auxiliary process

$$
K_{t}=\mathbf{m} t+\sigma B_{t}^{(e)}+\int_{0}^{t} \int_{(-1,1)} z \tilde{N}^{(e)}(\mathrm{d} s, \mathrm{~d} z)+\int_{0}^{t} \int_{\mathbb{R} \backslash(-1,1)} z N^{(e)}(\mathrm{d} s, \mathrm{~d} z),
$$

where

$$
\mathbf{m}=\alpha-\psi^{\prime}(0+)-\frac{\sigma^{2}}{2}-\int_{(-1,1)}\left(e^{z}-1-z\right) \pi(\mathrm{d} z) .
$$

It is important to note that when $\phi=0$, conditioned on the environment $\mathrm{K}$, the process $\mathrm{Z}$ satisfies the branching property. This is inherited from the branching property of the original $\mathrm{CB}$-process and the fact that the additional jumps are multiplicative. The proof of this fact is the same as in the Brownian environment case (see Theorem 1 in [32]).

Proposition 2 Suppose that $(\mathbf{H})$ holds. Then for every $z, \lambda, t>0$, a.s.

$$
\mathbb{E}_{z}\left[\exp \left\{-\lambda Z_{t} e^{-K_{t}}\right\} \mid K\right]=\exp \left\{-z v_{t}(0, \lambda, K)-\int_{0}^{t} \phi\left(v_{t}(r, \lambda, K) e^{-K_{r}}\right) \mathrm{d} r\right\}
$$

where for every $t, \lambda \geq 0$, the function $\left(v_{t}(s, \lambda, K), s \leq t\right)$ is the a.s. unique solution of the backward differential equation

$$
\frac{\partial}{\partial s} v_{t}(s, \lambda, K)=e^{K_{s}} \psi_{0}\left(v_{t}(s, \lambda, K) e^{-K_{s}}\right), \quad v_{t}(t, \lambda, K)=\lambda,
$$

and

$$
\psi_{0}(\lambda)=\psi(\lambda)-\lambda \psi^{\prime}(0)=\gamma^{2} \lambda^{2}+\int_{(0, \infty)}\left(e^{-\lambda x}-1+\lambda x\right) \mu(\mathrm{d} x), \quad \lambda \geq 0 .
$$

Proof The first part of the proof follows similar arguments as those used in Bansaye et al. [3]. The main problem in proving our result is finding the a.s. unique solution of the backward differential equation (12) in the general case. In order to do so, we need an approximation technique based on the Lévy-Itô decomposition of the Lévy process $K$. The proof of the latter can be found in Lemma 2 in the Appendix.

For sake of completeness, we remain the main steps of the proof which are similar as those used in [3]. We first define $\widetilde{Z}_{t}=Z_{t} e^{-K_{t}}$, for $t \geq 0$, and choose

$$
F(s, x)=\exp \left\{-x v_{t}(s, \lambda, K)-\int_{s}^{t} \phi\left(v_{t}(r, \lambda, K) e^{-K_{r}}\right) \mathrm{d} r\right\}, \quad s, x \geq 0,
$$

where $v_{t}(s, \lambda, K)$ is differentiable with respect to the variable $s$, non-negative and such that $v_{t}(t, \lambda, K)=\lambda$ for all $\lambda \geq 0$. We observe that conditioned on $K$, that $\left(F\left(s, \widetilde{Z}_{s}\right), s \leq t\right)$ is a martingale (using Itô's formula) if and only if

$$
\frac{\partial}{\partial s} v_{t}(s, \lambda, K)=\gamma^{2} v_{t}(s, \lambda, K)^{2} e^{-K_{s}}+e^{K_{s}} \int_{0}^{\infty}\left(e^{-e^{-K_{s}} v_{t}(s, \lambda, K) z}-1+e^{-K_{s}} v_{t}(s, \lambda, K) z\right) \mu(\mathrm{d} z)
$$


That is $v_{t}(s, \lambda, K)$ solves (12). Providing that $v_{t}(s, \lambda, K)$ exist a.s., we see that the process $\left(\exp \left\{-\widetilde{Z}_{s} v_{t}(s, \lambda, K)\right\}, 0 \leq s \leq t\right)$ conditioned on $K$ is a martingale, and hence

$$
\mathbb{E}_{z}\left[\exp \left\{-\lambda \widetilde{Z}_{t}\right\} \mid K\right]=\exp \left\{-z v_{t}(0, \lambda, K)-\int_{0}^{t} \phi\left(v_{t}(r, \lambda, K) e^{-K_{r}}\right) \mathrm{d} r\right\}
$$

It is important to note that if $\left|\psi^{\prime}(0+)\right|=\infty$, the auxiliary process can be taken as

$$
K_{t}^{(0)}=\mathbf{n} t+\sigma B_{t}^{(e)}+\int_{0}^{t} \int_{(-1,1)} z \tilde{N}^{(e)}(\mathrm{d} s, \mathrm{~d} z)+\int_{0}^{t} \int_{\mathbb{R} \backslash(-1,1)} z N^{(e)}(\mathrm{d} s, \mathrm{~d} z),
$$

where $\mathbf{n}=\alpha-\frac{\sigma^{2}}{2}-\int_{(-1,1)}\left(e^{z}-1-z\right) \pi(\mathrm{d} z)$. Following the same arguments as in the previous proposition and replacing $K$ with $K^{(0)}$, one can deduce that $v_{t}\left(s, \lambda, K^{(0)}\right)$ is the unique solution to the backward differential equation

$$
\frac{\partial}{\partial s} v_{t}\left(s, \lambda, K^{(0)}\right)=e^{K_{s}^{(0)}} \psi\left(v_{t}\left(s, \lambda, K^{(0)}\right) e^{-K_{s}^{(0)}}\right), \quad v_{t}\left(t, \lambda, K^{(0)}\right)=\lambda,
$$

whenever this equation has a solution.

The latter observation allows us to compute explicitly the Laplace exponent of the so called Neveu case. Recall that the Neveu branching process in a Lévy random environment has branching mechanism given by

$$
\psi(u)=u \log (u)=c u+\int_{(0, \infty)}\left(e^{-u x}-1+u x \mathbf{1}_{\{x<1\}}\right) x^{-2} \mathrm{~d} x, \quad u>0,
$$

where $c \in \mathbb{R}$ is a suitable constant. In this particular case, the backward differential equation (13) satisfies

$$
\frac{\partial}{\partial s} v_{t}\left(s, \lambda, K^{(0)}\right)=v_{t}(s, \lambda, \delta) \log \left(e^{-K_{s}^{(0)}} v_{t}\left(s, \lambda, K^{(0)}\right), \quad v_{t}\left(t, \lambda, K^{(0)}\right)=\lambda .\right.
$$

The above equation has a solution and is given by

$$
v_{t}\left(s, \lambda, K^{(0)}\right)=\exp \left\{e^{s}\left(\int_{s}^{t} e^{-u} K_{u}^{(0)} \mathrm{d} u+\log (\lambda) e^{-t}\right)\right\} .
$$

Hence, for all $z, \lambda, t>0$, we deduce

$$
\mathbb{E}_{z}\left[\exp \left\{-\lambda Z_{t} e^{-K_{t}^{(0)}}\right\} \mid K^{(0)}\right]=\exp \left\{-z \lambda^{e^{-t}} \exp \left\{\int_{0}^{t} e^{-s} K_{s}^{(0)} \mathrm{d} s\right\}\right\} \quad \text { a.s. }
$$

By taking limits as $\lambda$ goes to $\infty$ in the previous expression and then taking expectation, we obtain $\mathbb{P}_{z}\left(Z_{t}>0\right)=1$, for $t \geq 0$. By integration by parts, we deduce

$$
\int_{0}^{t} e^{-s} K_{s}^{(0)} \mathrm{d} s=-e^{-t} K_{t}^{(0)}+\int_{0}^{t} e^{-s} \mathrm{~d} K_{s}^{(0)}
$$

Under the assumption $\mathbb{E}\left[\left|K_{1}^{(0)}\right|\right]<\infty$, we deduce from Theorem 17.5 in Sato [36], that the r.v. $\int_{0}^{\infty} e^{-s} K_{s}^{(0)} \mathrm{d} s=\int_{0}^{\infty} e^{-s} \mathrm{~d} K_{s}^{(0)}$ is self-decomposable with characteristic exponent given 
by $\psi(\lambda)=\int_{0}^{\infty} \psi_{K^{(0)}}\left(\lambda e^{-s}\right) \mathrm{d} s$, where $\psi_{K^{(0)}}$ denotes the characteristic exponent of $K^{(0)}$. Therefore, if we take limits as $t \uparrow \infty$ in (14), we observe that for $z, \lambda>0$

$$
\mathbb{E}_{z}\left[\exp \left\{-\lambda \lim _{t \rightarrow \infty} Z_{t} e^{-K_{t}^{(0)}}\right\} \mid K^{(0)}\right]=\exp \left\{-z \exp \left\{\int_{0}^{\infty} e^{-s} \mathrm{~d} K_{s}^{(0)}\right\}\right\} .
$$

Since the right-hand side of the above identity does not depend on $\lambda$, this implies that

$$
\mathbb{P}_{z}\left(\lim _{t \rightarrow \infty} Z_{t} e^{-K_{t}^{(0)}}=0\right)=\mathbb{E}\left[\exp \left\{-z \exp \left\{\int_{0}^{\infty} e^{-s} \mathrm{~d} K_{s}^{(0)}\right\}\right\}\right]
$$

In conclusion, the Neveu process in Lévy environment survives a.s. but when the Lévy process $K^{(0)}$ does not drift to $\infty$, the extinction probability is given by the previous expression.

Another interesting example is the self-similar CB-processes in a Lévy random environment. In this example the branching mechanism is taken as follows

$$
\psi(\lambda)=c \lambda^{\alpha}, \quad \lambda \geq 0,
$$

for some $\alpha \in(0,1) \cup(1,2]$ and $c \in \mathbb{R}$ such that $c(\alpha-1)>0$. If $\alpha=2$, we observe that $\mu(0, \infty)=0$ and $c=\gamma^{2}$. In the case $\alpha \in(0,1) \cup(1,2)$, the process $Z$ satisfies the SDE

$$
Z_{t}=Z_{0}+\int_{0}^{t} Z_{s-} \mathrm{d} S_{s}+\int_{0}^{t} \int_{0}^{\infty} \int_{0}^{Z_{s-}} z \widehat{N}(\mathrm{~d} s, \mathrm{~d} z, \mathrm{~d} u)
$$

where $N$ is a Poisson random measure with intensity

$$
\frac{c \alpha(\alpha-1)}{\Gamma(2-\alpha)} \frac{1}{z^{1+\alpha}} \mathrm{d} s \mathrm{~d} z \mathrm{~d} u
$$

$\tilde{N}$ is its compensated version and $\widehat{N}=N \mathbf{1}_{\{\alpha \in(0,1)\}}+\tilde{N} \mathbf{1}_{\{\alpha \in(1,2)\}}$. Note that

$$
\psi^{\prime}(0+)= \begin{cases}-\infty & \text { if } \alpha \in(0,1) \\ 0 & \text { if } \alpha \in(1,2]\end{cases}
$$

Hence, when $\alpha \in(1,2]$, we have $K_{t}=K_{t}^{(0)}$, for $t \geq 0$. We use the backward differential equation (13) and observe that it satisfies

$$
\frac{\partial}{\partial s} v_{t}\left(s, \lambda, K^{(0)}\right)=c v_{t}^{\alpha}\left(s, \lambda, K^{(0)}\right) e^{-(\alpha-1) K_{s}^{(0)}}
$$

Assuming that $v_{t}\left(t, \lambda, K^{(0)}\right)=\lambda$, we can solve the above equation and after some straightforward computations, we get

$$
v_{t}\left(s, \lambda, K^{(0)}\right)=\left(\lambda^{1-\alpha}+(\alpha-1) c \int_{s}^{t} e^{-(\alpha-1) K_{u}^{(0)}} \mathrm{d} u\right)^{-1 /(\alpha-1)}
$$

Hence, from (11) the following identity holds a.s.

$$
\mathbb{E}_{z}\left[\exp \left\{-\lambda Z_{t} e^{-K_{t}^{(0)}}\right\} \mid K^{(0)}\right]=\exp \left\{-z\left(\lambda^{1-\alpha}+(\alpha-1) c \int_{0}^{t} e^{-(\alpha-1) K_{u}^{(0)}} \mathrm{d} u\right)^{-1 /(\alpha-1)}\right\} .
$$


Observe that the probabilities of survival and non-explosion can be determined explicitly in terms of the exponential functional $\int_{0}^{t} e^{-(\alpha-1) K_{u}^{(0)}} \mathrm{d} u$, by taking $\lambda$ goes to $\infty$ or $\lambda$ goes to 0 , respectively. In other words, for all $z>0$

$$
\mathbb{P}_{z}\left(Z_{t}>0 \mid K^{(0)}\right)=1-\mathbf{1}_{\{\alpha>1\}} \exp \left\{-z\left((\alpha-1) c \int_{0}^{t} e^{-(\alpha-1) K_{u}^{(0)}} \mathrm{d} u\right)^{-1 /(\alpha-1)}\right\}, \quad \text { a.s. },
$$

and

$$
\mathbb{P}_{z}\left(Z_{t}<\infty \mid K^{(0)}\right)=\mathbf{1}_{\{\alpha>1\}}+\mathbf{1}_{\{\alpha<1\}} \exp \left\{-z\left((\alpha-1) c \int_{0}^{t} e^{-(\alpha-1) K_{u}^{(0)}} \mathrm{d} u\right)^{-1 /(\alpha-1)}\right\}, \quad \text { a.s. }
$$

The asymptotic behaviour of these probabilities has been computed recently and independently by $\mathrm{Li}$ and $\mathrm{Xu}$ [28] and by Palau et al. [33]. In particular, and similarly to the results obtained by Bansaye et al. [3] and Palau and Pardo [32], the authors in [28] and [33] obtained five different regimes for the probability of survival when $\alpha \in(1,2]$ and the authors in [33] obtained three different regimes for the probability of non-explosion when $\alpha \in(0,1)$. All the regimens depend on the characteristic of the Lévy process $K^{(0)}$.

\subsection{Long Term Behaviour of CB-Processes in a Lévy Random Environment}

In the sequel, we exclude from our model the competition mechanism $\beta$ and the immigration term $M^{(i m)}$. In this section, we are interested in determining the long term behaviour of CBprocesses in a Lévy random environment (CBLRE for short). Our methodology follows similar arguments as those used in Proposition 2 in [32] and Corollary 2 in [3].

Let denote by $\Psi_{K}$ the characteristic exponent of the Lévy process $K$, i.e.

$$
\Psi_{K}(\theta)=-\log \mathbb{E}\left[e^{i \theta K_{1}}\right] \quad \text { for } \theta \in \mathbb{R}
$$

We introduce the functions

$$
\Phi(\lambda)=\lambda^{-1} \psi_{0}(\lambda) \text { for } \lambda \geq 0
$$

and

$$
A(x)=\mathbf{m}+\pi((1, \infty))+\int_{1}^{x} \pi((y, \infty)) \mathrm{d} y, \quad \text { for } x>0 .
$$

Proposition 3 Assume that $(\mathbf{H})$ holds. Let $\left(Z_{t}, t \geq 0\right)$ be a CBLRE with branching mechanism given by $\psi$, and $z>0$

(i) If the process $K$ drifts to $-\infty$, then $\mathbb{P}_{z}\left(\lim _{t \rightarrow \infty} Z_{t}=0 \mid K\right)=1$, a.s.

(ii) If the process $K$ oscillates, then $\mathbb{P}_{z}\left(\liminf _{t \rightarrow \infty} Z_{t}=0 \mid K\right)=1$, a.s. Moreover if $\gamma>0$ then

$$
\mathbb{P}_{z}\left(\lim _{t \rightarrow \infty} Z_{t}=0 \mid K\right)=1, \quad \text { a.s. }
$$

(iii) If the process $K$ drifts to $+\infty$, so that $A(x)>0$ for all $x$ larger enough. Then if

$$
\int_{(a, \infty)} \frac{x}{A(x)}\left|\mathrm{d} \Phi\left(e^{-x}\right)\right|<\infty, \quad \text { for some } a>0,
$$


we have $\mathbb{P}_{z}\left(\liminf _{t \rightarrow \infty} Z_{t}>0 \mid K\right)>0$ a.s., and there exists a non-negative finite r.v. $W$ such that

$$
Z_{t} e^{-K_{t}} \underset{t \rightarrow \infty}{\longrightarrow} W, \quad \text { a.s } \quad \text { and } \quad\{W=0\}=\left\{\lim _{t \rightarrow \infty} Z_{t}=0\right\} .
$$

In particular, if $0<\mathbb{E}\left[K_{1}\right]<\infty$, then the above integral condition is equivalent to

$$
\int^{\infty} x \ln x \mu(\mathrm{d} x)<\infty
$$

Proof Parts (i) and (ii) follow from the same arguments used in the proof of Proposition 2 in [32], so we skip their proofs. The key part is to note that via Ito calculus, $Z_{t} e^{-K_{t}}$ is a non-negative local martingale. Therefore $Z_{t} e^{-K_{t}}$ is a non-negative supermartingale that converges a.s. to a non-negative finite random variable, here denoted by $W$.

Now, we prove part (iii). We first observe that $v_{t}(\cdot, \lambda, K)$, the a.s. solution to the backward differential equation (12), is non-decreasing on $[0, t]$ (since $\psi_{0}$ is positive). Thus for all $s \in[0, t], v_{t}(s, \lambda, K) \leq \lambda$. Observe that $\Phi(0)=\psi_{0}^{\prime}(0+)=0$ and since $\psi_{0}$ is convex, we deduce that $\Phi$ is increasing. Hence

$$
\frac{\partial}{\partial s} v_{t}(s, \lambda, K)=v_{t}(s, \lambda, K) \Phi\left(v_{t}(s, \lambda, K) e^{-K_{s}}\right) \leq v_{t}(s, \lambda, K) \Phi\left(\lambda e^{-K_{s}}\right) .
$$

Therefore, for every $s \leq t$, we have

$$
v_{t}(s, \lambda, K) \geq \lambda \exp \left\{-\int_{s}^{t} \Phi\left(\lambda e^{-K_{s}}\right) \mathrm{d} s\right\} .
$$

In particular,

$$
\liminf _{t \rightarrow \infty} v_{t}(0, \lambda, K) \geq \lambda \exp \left\{-\int_{0}^{\infty} \Phi\left(\lambda e^{-K_{s}}\right) \mathrm{d} s\right\} .
$$

If the integral on the right-hand side is a.s. finite, then

$$
\liminf _{t \rightarrow \infty} v_{t}(0, \lambda, K) \geq \lambda \exp \left\{-\int_{0}^{\infty} \Phi\left(\lambda e^{-K_{s}}\right) \mathrm{d} s\right\}>0, \quad \text { a.s. }
$$

implying

$$
\mathbb{E}_{z}\left[e^{-\lambda W} \mid K\right] \leq \exp \left\{-z \lambda \exp \left\{-\int_{0}^{\infty} \Phi\left(\lambda e^{-K_{s}}\right) \mathrm{d} s\right\}\right\}<1, \quad \text { a.s. }
$$

and in particular $\mathbb{P}_{z}\left(\liminf _{t \rightarrow \infty} Z_{t}>0 \mid K\right)>0$ a.s. Next, we use Lemma 20 in [3] and the branching property of $Z$, to deduce

$$
\{W=0\}=\left\{\lim _{t \rightarrow \infty} Z_{t}=0\right\} .
$$

In order to finish our proof, we show that the integral condition (15) implies

$$
\int_{0}^{\infty} \Phi\left(\lambda e^{-K_{s}}\right) \mathrm{d} s<\infty \quad \text { a.s. }
$$


We first introduce $\varsigma=\sup \left\{t \geq 0: K_{t} \leq 0\right\}$ and observe

$$
\int_{0}^{\infty} \Phi\left(\lambda e^{-K_{s}}\right) \mathrm{d} s=\int_{0}^{\varsigma} \Phi\left(\lambda e^{-K_{s}}\right) \mathrm{d} s+\int_{\varsigma}^{\infty} \Phi\left(\lambda e^{-K_{s}}\right) \mathrm{d} s
$$

Since $\varsigma<\infty$ a.s., the first integral of the right-hand side is a.s. finite. For the second integral, we use Theorem 1 in Erickson and Maller [16] which assures us that

$$
\int_{\varsigma}^{\infty} \Phi\left(\lambda e^{-K_{s}}\right) \mathrm{d} s<\infty, \quad \text { a.s. }
$$

if the integral condition (15) holds.

Finally, we assume that $0 \leq \mathbb{E}\left[K_{1}\right]<\infty$ and observe that $\lim _{x \rightarrow \infty} A(x)$ is finite. In particular, this implies that the integral condition (15) is equivalent to

$$
\int_{0}^{\infty} \Phi\left(\lambda e^{-y}\right) \mathrm{d} y<\infty
$$

Moreover, we have

$$
\begin{aligned}
\int_{0}^{\infty} \Phi\left(\lambda e^{-y}\right) \mathrm{d} y=\int_{0}^{\lambda} \frac{\Phi(\theta)}{\theta} \mathrm{d} \theta & =\gamma^{2} \lambda+\int_{0}^{\lambda} \frac{\mathrm{d} \theta}{\theta^{2}} \int_{(0, \infty)}\left(e^{-\theta x}-1+\theta x\right) \mu(\mathrm{d} x) \\
& =\gamma^{2} \lambda+\int_{(0, \infty)} \mu(\mathrm{d} x) \int_{0}^{\lambda}\left(e^{-\theta x}-1+\theta x\right) \frac{\mathrm{d} \theta}{\theta^{2}} \\
& =\gamma^{2} \lambda+\int_{(0, \infty)} x\left(\int_{0}^{\lambda x}\left(e^{-y}-1+y\right) \frac{\mathrm{d} y}{y^{2}}\right) \mu(\mathrm{d} x) .
\end{aligned}
$$

Since the function

$$
g_{\lambda}(x)=\int_{0}^{\lambda x}\left(e^{-y}-1+y\right) \frac{\mathrm{d} y}{y^{2}},
$$

is equivalent to $\lambda x / 2$ as $x \rightarrow 0$ and equivalent to $\ln x$ as $x \rightarrow \infty$, we deduce that

$$
\int_{0}^{\infty} \Phi\left(\lambda e^{-y}\right) \mathrm{d} y<\infty \text { if and only if } \int^{\infty} x \ln x \mu(\mathrm{d} x)<\infty .
$$

Now, we derive a central limit theorem in the supercritical regime which follows from Theorem 3.5 in Doney and Maller [15] and similar arguments as those used in Corollary 3 in [3], so we skip its proof.

For $x>0$, let

$$
T(x)=\pi((x, \infty))+\pi((-\infty,-x)) \quad \text { and } \quad U(x)=\sigma^{2}+\int_{0}^{x} y T(y) \mathrm{d} y
$$

Corollary 1 Assume that $K$ drifts to $+\infty, T(x)>0$ for all $x>0$, and (15) is satisfied. There are two measurable functions $a(t), b(t)>0$ such that, conditioned on $\{W>0\}$,

$$
\frac{\log \left(Z_{t}\right)-a(t)}{b(t)} \underset{t \rightarrow \infty}{\stackrel{d}{\longrightarrow}} \mathcal{N}(0,1),
$$


if and only if

$$
\frac{U(x)}{x^{2} T(x)} \rightarrow \infty \quad \text { as } x \rightarrow \infty
$$

where $\stackrel{d}{\rightarrow}$ means convergence in distribution and $\mathcal{N}(0,1)$ denotes a centred Gaussian random variable with variance equals 1 .

It is important to note that if $\int_{\{|x|>1\}} x^{2} \pi(\mathrm{d} x)<\infty$, then for all $t>0$,

$$
a(t):=\left(\mathbf{m}+\int_{\{|x| \geq 1\}} x \pi(\mathrm{d} x)\right) t \quad \text { and } \quad b^{2}(t):=\left(\sigma^{2}+\int_{\mathbb{R}} x^{2} \pi(\mathrm{d} x)\right) t,
$$

which is similar to the result obtained in Corollary 3 in [3].

\subsection{Population Model with Competition in a Lévy Random Environment}

We now study an extension of the competition model given in Evans et al. [17]. In this model, we exclude the immigration term and take the branching and competition mechanisms as follows

$$
\beta(x)=k x^{2} \quad \text { and } \quad \psi(\lambda)=a \lambda \quad \text { for } x, \lambda \geq 0,
$$

where $k$ is a positive constant. Hence, we define a branching process in a Lévy random environment process $\left(Z_{t}, t \geq 0\right)$ as the solution of the SDE

$$
Z_{t}=Z_{0}+\int_{0}^{t} Z_{s}\left(a-k Z_{s}\right) \mathrm{d} s+\int_{0}^{t} Z_{s-} \mathrm{d} S_{s}
$$

where the environment is the Lévy process given in (9).

From Theorem 1, there is a unique non negative strong solution of (16) satisfying the Markov property. Moreover, we have the following result that in particular says that the process $Z$ is the inverse of a generalised Ornstein-Uhlenbeck process.

Proposition 4 Suposse that $\left(Z_{t}, t \geq 0\right)$ is the unique strong solution of (16). Then, it satisfies

$$
Z_{t}=\frac{Z_{0} e^{K_{t}}}{1+k Z_{0} \int_{0}^{t} e^{K_{s}} \mathrm{~d} s}, \quad t \geq 0,
$$

where $K$ is the Lévy process defined in (10). Moreover, if $Z_{0}=z>0$ then, $Z_{t}>0$ for all $t \geq 0$ a.s. and it has the following asymptotic behaviour:

(i) If the process $K$ drifts to $-\infty$, then $\lim _{t \rightarrow \infty} Z_{t}=0$ a.s.

(ii) If the process $K$ oscillates, then $\liminf _{t \rightarrow \infty} Z_{t}=0$ a.s.

(iii) If the process $K$ drifts to $\infty$, then $\left(Z_{t}, t \geq 0\right)$ has a stationary distribution whose density satisfies for $z>0$,

$$
\mathbb{P}_{z}\left(Z_{\infty} \in \mathrm{d} x\right)=h\left(\frac{1}{k x}\right) \frac{\mathrm{d} x}{x^{2}}, \quad x>0
$$

where

$$
\int_{t}^{\infty} h(x) \mathrm{d} x=\int_{\mathbb{R}} h\left(t e^{-y}\right) U(\mathrm{~d} y), \quad \text { a.e. } t \text { on }(0, \infty),
$$


and $U$ denotes the potential measure associated to $K$, i.e.

$$
U(\mathrm{~d} x)=\int_{0}^{\infty} \mathbb{P}\left(K_{s} \in \mathrm{d} x\right) \mathrm{d} s, \quad x \in \mathbb{R} .
$$

Moreover, if $0<\mathbb{E}\left[K_{1}\right]<\infty$, then

$$
\lim _{t \rightarrow \infty} \frac{1}{t} \int_{0}^{t} Z_{s} \mathrm{~d} s=\frac{1}{k} \mathbb{E}\left[K_{1}\right], \quad \text { a.s. }
$$

and for every measure function $f: \mathbb{R}_{+} \rightarrow \mathbb{R}_{+}$we have

$$
\lim _{t \rightarrow \infty} \frac{1}{t} \int_{0}^{t} f\left(Z_{s}\right) \mathrm{d} s=\mathbb{E}\left[f\left(\frac{1}{k I_{\infty}(-K)}\right)\right], \quad \text { a.s. }
$$

where $I_{\infty}(-K)=\int_{0}^{\infty} e^{-K_{s}} \mathrm{~d} s$.

Proof By Itô's formula, we see that the process $Z$ satisfies (17). Moreover, since the Lévy process $K$ has infinite lifetime, then we necessarily have $Z_{t}>0$ a.s.

Now in order to describe the asymptotic behaviour of $Z$ we recall the following result of Bertoin and Yor [9] on exponential functionals of Lévy processes,

$$
\int_{0}^{\infty} e^{K_{s}} \mathrm{~d} s<\infty \quad \text { a.s. if and only if } K \text { drifts to }-\infty
$$

Therefore part (i) follows directly from (17) and (18). Next, we prove part (ii). Assume that $K$ oscillates. On the one hand, we have

$$
Z_{t}=\frac{Z_{0}}{e^{-K_{t}}+k Z_{0} e^{-K_{t}} \int_{0}^{t} e^{K_{s}} \mathrm{~d} s} \leq \frac{1}{k e^{-K_{t}} \int_{0}^{t} e^{K_{s}} \mathrm{~d} s} .
$$

On the other hand, the Duality Lemma (see for instance Lemma 3.4 in [23]) tells us that $\left\{K_{(t-s)-}-K_{t}: 0 \leq s \leq t\right\}$ and $\left\{-K_{s}: 0 \leq s \leq t\right\}$ have the same law under $\mathbb{P}$. Then, we deduce

$$
\left(K_{t}, e^{-K_{t}} \int_{0}^{t} e^{K_{s}} \mathrm{~d} s\right) \text { is equal in law to }\left(K_{t}, \int_{0}^{t} e^{-K_{s}} \mathrm{~d} s\right) .
$$

From (18) and our assumption, we have that the exponential functional of $K$ goes to $\infty$ as $t \rightarrow \infty$. This implies that $\lim _{t \rightarrow \infty} Z_{t}=0$ in distribution and therefore,

$$
\liminf _{t \rightarrow \infty} Z_{t}=0, \quad \text { a.s. }
$$

Finally, we assume that the process $K$ drifts to $\infty$. Then, form the previous observation, $Z_{t}$ is equal in law to

$$
Z_{t} \stackrel{d}{=} \frac{Z_{0}}{e^{-K_{t}}+k Z_{0} \int_{0}^{t} e^{-K_{s}} \mathrm{~d} s} .
$$

Using (18), we have that $Z_{t}$ converges in distribution to

$$
\left(k \int_{0}^{\infty} e^{-K_{s}} \mathrm{~d} s\right)^{-1}
$$

The form of the density follows from Theorem 1 of Arista and Rivero [2]. 
Now, observe that

$$
\int_{0}^{t} Z_{s} \mathrm{~d} s=\frac{1}{k} \ln \left(1+k Z_{0} \int_{0}^{t} e^{K_{s}} \mathrm{~d} s\right) .
$$

Therefore if $0<\mathbb{E}\left[K_{1}\right]<\infty$, an application of the law of large numbers allow us to deduce

$$
\lim _{t \rightarrow \infty} \frac{1}{t} \int_{0}^{t} Z_{s} \mathrm{~d} s=\lim _{t \rightarrow \infty} \frac{1}{k t} \ln \left(\int_{0}^{t} e^{K_{s}} \mathrm{~d} s\right)=\frac{1}{k} \mathbb{E}\left[K_{1}\right], \quad \text { a.s. }
$$

In order to prove the last assertion of our proposition, let us introduce $X^{(x)}=\left(X_{t}^{(x)}, t \geq 0\right)$ the positive self-similar Markov process associated to $K$ via the Lamperti transform with scaling index 1 . That is to say, for all $x>0$

$$
X_{t}^{(x)}=x e^{\left.K_{\tau(t x}-1\right)}, \quad t \geq 0,
$$

where the time change $\tau$ is defined as follows

$$
\tau(t)=\inf \left\{s \geq 0: \int_{0}^{s} e^{K_{r}} d r>t\right\} .
$$

This process satisfies the scaling property, i.e. for $a>0$, the following identity in law follows

$$
\left(a X_{t}^{(x)}, t \geq 0\right) \stackrel{d}{=}\left(X_{a t}^{(a x)}, t \geq 0\right) .
$$

Next, we define the process $Y$ as follows

$$
Y_{t}^{(x)}:=e^{-k t} X_{\left(e^{k t}-1\right) / k}^{(x)}, \quad t \geq 0,
$$

By the scaling property, it turns out to have the same law as

$$
\left(X_{\left(1-e^{-k t}\right) / k}^{\left(x e^{-k t}\right)}, t \geq 0\right) .
$$

Since $0<\mathbb{E}\left[K_{1}\right]<\infty$, Theorem 1 of Bertoin and Yor in [8] tells us that for all $x>0$, $Y_{t}^{(x)} \rightarrow X_{1 / k}^{(0)}$ as $t \rightarrow \infty$, and for any measurable function $f: \mathbb{R}_{+} \longrightarrow \mathbb{R}_{+}$

$$
\mathbb{E}\left[f\left(X_{1 / k}^{(0)}\right)\right]:=\int_{(0, \infty)} f(x) \mu(\mathrm{d} x)=\frac{1}{\mathbb{E}\left[K_{1}\right]} \mathbb{E}\left[f\left(\frac{1}{k I_{\infty}(-K)}\right) \frac{1}{I_{\infty}(-K)}\right],
$$

where $I_{\infty}(-K)=\int_{0}^{\infty} e^{-K_{s}} \mathrm{~d} s$. Then, $Y$ is a Markov process with invariant distribution $\mu$. Moreover, by the Ergodic Theorem

$$
\lim _{t \rightarrow \infty} \frac{1}{t} \int_{0}^{t} f\left(Y_{s}^{(x)}\right) \mathrm{d} s=\int_{(0, \infty)} f(x) \mu(\mathrm{d} x) .
$$

In one hand, observe that by the definition of $Y$ and $\tau$,

$$
Y_{t}^{\left(Z_{0}\right)}=Z_{0} e^{-k t} e^{K_{\tau\left(\left(e^{k t}-1\right) /\left(k Z_{0}\right)\right)}}=\left.\frac{Z_{0} e^{K_{v}}}{1+k Z_{0} \int_{0}^{v} e^{K_{s}} \mathrm{~d} s}\right|_{v=\tau\left(\left(e^{k t}-1\right) /\left(k Z_{0}\right)\right)}=Z_{\tau\left(\left(e^{k t}-1\right) /\left(k Z_{0}\right)\right)} .
$$

On the other hand, by identity (19), we deduce for all $t \geq 0$

$$
\tau\left(\frac{e^{k t}-1}{k Z_{0}}\right)=\inf \left\{s>0: \int_{0}^{s} Z_{r} \mathrm{~d} r>t\right\} .
$$


This implies

$$
\int_{0}^{t} f\left(Z_{s}\right) \mathrm{d} s=\int_{0}^{\tau\left(\frac{e^{k t}-1}{k Z_{0}}\right)} f\left(Z_{\tau\left(\frac{e^{k s-1}}{k Z_{0}}\right)}\right) \frac{1}{Z_{\tau\left(\frac{e^{k s}-1}{k Z_{0}}\right)}} \mathrm{d} s=\int_{0}^{\tau\left(\frac{e^{k t}-1}{k Z_{0}}\right)} f\left(Y_{s}^{\left(Z_{0}\right)}\right) \frac{1}{Y_{s}^{\left(Z_{0}\right)}} \mathrm{d} s .
$$

By taking $f(x)=x$ in the previous equality, we get

$$
\lim _{t \rightarrow \infty} \frac{\tau\left(\frac{e^{k t}-1}{k Z_{0}}\right)}{t}=\lim _{t \rightarrow \infty} \frac{1}{t} \int_{0}^{t} Z_{s} \mathrm{~d} s .
$$

Putting all the pieces together, i.e. by (20), (21), (22) and (23), we have

$$
\lim _{t \rightarrow \infty} \frac{1}{t} \int_{0}^{t} f\left(Z_{s}\right) \mathrm{d} s=\mathbb{E}\left[f\left(\frac{1}{k I_{\infty}(-K)}\right)\right], \quad \text { a.s. }
$$

This completes the proof.

The asymptotic behaviour of the positive moments of $Z_{t}$ has been studied in Palau et al. [33] using a fine study of the negative moments of exponential functional of Lévy processes. In particular four different regimes appears that depends on the characteristic of the Lévy process $K$.

We finish this section with two important observations in two particular cases. We first assume that the process $K$ drifts to $+\infty$ and that satisfies

$$
\int_{[1, \infty)} e^{q x} \pi(\mathrm{d} x)<\infty \quad \text { for every } q>0
$$

i.e. that has exponential moments of all positive orders. In this situation, the characteristic exponent $\Psi_{K}$ has an analytic extension to the half-plane with negative imaginary part, and one has

$$
\mathbb{E}\left[e^{q K_{t}}\right]=e^{t \psi_{K}(q)}<\infty, \quad t, q \geq 0,
$$

where $\psi_{K}(q)=-\Psi_{K}(-i q)$ for $q \geq 0$. Hence, according to Theorem 3 in Bertoin and Yor [9] the stationary distribution has positive moments and satisfies, for $z>0$ and $n \geq 1$,

$$
\mathbb{E}_{z}\left[Z_{\infty}^{n}\right]=\psi_{K}^{\prime}(0+) \frac{\psi_{K}(1) \cdots \psi_{K}(n-1)}{(n-1) !}
$$

Finally, we assume that the process $K$ drifts to $-\infty$ and has no negative jumps. Observe that the process $Z$ inherited the latter property and we let $Z_{0}=z>0$. Under this assumption, we can compute the Laplace transform of the first passage time from below a level $z>b>0$ of the process $Z$, i.e.

$$
\sigma_{b}=\inf \left\{s \geq 0: Z_{s} \leq b\right\} .
$$

In this situation, the characteristic exponent $\Psi_{k}$ has an analytic extension to the half-plane with positive imaginary part, and one has

$$
\mathbb{E}\left[e^{-q K_{t}}\right]=e^{t \hat{\psi}_{K}(q)}<\infty, \quad t, q \geq 0,
$$


where $\hat{\psi}_{K}(q)=-\Psi_{K}(i q)$ for $q \geq 0$. Define, for all $t \geq 0, \mathcal{F}_{t}=\sigma\left(K_{s}: s \leq t\right)$ and consider the Esscher transform

$$
\left.\frac{\mathrm{d} \mathbb{P}^{\kappa(\lambda)}}{\mathrm{d} \mathbb{P}}\right|_{\mathcal{F}_{t}}=e^{-\kappa(\lambda) K_{t}-\lambda t}, \quad \text { for } \lambda \geq 0
$$

where $\kappa(\lambda)$ is the largest solution to $\hat{\psi}_{K}(u)=\lambda$. Under $\mathbb{P}^{\kappa(\lambda)}$, the process $K$ is still a spectrally positive and its Laplace exponent, $\hat{\psi}_{\kappa(\lambda)}$ satisfies the relation

$$
\hat{\psi}_{\kappa(\lambda)}(u)=\hat{\psi}_{K}(\kappa(\lambda)+u)-\lambda, \quad \text { for } u \geq 0 .
$$

See [23, Chap. 8] for further details on the above remarks. Note in particular that it is easy to verify that $\hat{\psi}_{\kappa(\lambda)}^{\prime}(0+)>0$ and hence the process $K$ under $\mathbb{P}^{\kappa(\lambda)}$ drifts to $-\infty$. According to earlier discussion, this guarantees that also under $\mathbb{P}^{\kappa(\lambda)}$, the process $Z$ goes to 0 as $t \rightarrow \infty$.

Lemma 1 Suppose that $\lambda \geq 0$ and that $\kappa(\lambda)>1$, then for all $0<b \leq z$,

$$
\mathbb{E}_{z}\left[e^{-\lambda \sigma_{b}}\right]=\frac{\mathbb{E}^{\kappa(\lambda)}\left[\left(1+k z I_{\infty}(K)\right)^{\kappa(\lambda)}\right]}{\mathbb{E}^{\kappa(\lambda)}\left[\left(z b^{-1}+k z I_{\infty}(K)\right)^{\kappa(\lambda)}\right]},
$$

where

$$
I_{\infty}(K)=\int_{0}^{\infty} e^{K_{s}} \mathrm{~d} s
$$

Proof From the absence of negative jumps we have $Z_{\sigma_{b}}=b$ on the event $\left\{\sigma_{b}<\infty\right\}$ and in particular

$$
b=\frac{z e^{K_{\sigma_{b}}}}{1+k z \int_{0}^{\sigma_{b}} e^{K_{s}} \mathrm{~d} s} .
$$

On the other hand, from the Markov property and the above identity, we have

$$
1+k z I_{\infty}(K)=1+k z \int_{0}^{\sigma_{b}} e^{K_{s}} \mathrm{~d} s+k z e^{K_{\sigma_{b}}} \int_{0}^{\infty} e^{K_{\sigma_{b}+s}-K_{\sigma_{b}}} \mathrm{~d} s=e^{K_{\sigma_{b}}}\left(\frac{z}{b}+z k I_{\infty}^{\prime}\right),
$$

where $I_{\infty}^{\prime}$ is an independent copy of $I_{\infty}(K)$.

The latter identity and the Escher transform imply that for $\lambda \geq 0$

$$
\mathbb{E}_{z}\left[e^{-\lambda \sigma_{b}}\right]=\mathbb{E}^{\kappa(\lambda)}\left[e^{\kappa(\lambda) K_{\sigma_{b}}}\right]=\frac{\mathbb{E}^{\kappa(\lambda)}\left[\left(1+k z I_{\infty}(K)\right)^{\kappa(\lambda)}\right]}{\mathbb{E}^{\kappa(\lambda)}\left[\left(\frac{z}{b}+z k I_{\infty}(K)\right)^{\kappa(\lambda)}\right]},
$$

provided the quantity $\mathbb{E}^{\kappa(\lambda)}\left[\left(a+k z I_{\infty}(K)\right)^{\kappa(\lambda)}\right]$ is finite, for $a>0$.

Observe that for $s \geq 1$,

$$
\mathbb{E}^{\kappa(\lambda)}\left[\left(a+I_{\infty}(K)\right)^{s}\right] \leq 2^{s-1}\left(a^{s}+\mathbb{E}^{\kappa(\lambda)}\left[I_{\infty}(K)^{s}\right]\right),
$$

hence it suffices to investigate the finiteness of $\mathbb{E}^{\kappa(\lambda)}\left[I_{\infty}(K)^{s}\right]$. According to Lemma 2.1 in Maulik and Zwart [31] the expectation $\mathbb{E}^{\kappa(\lambda)}\left[I_{\infty}(K)^{s}\right]$ is finite for all $s \geq 0$ such that $-\hat{\psi}_{\kappa(\lambda)}(-s)>0$. Since $\hat{\psi}_{\kappa(\lambda)}(-s)$ is well defined for $\kappa(\lambda)-s \geq 0$, then a straightforward computation gives us that $\mathbb{E}^{\kappa(\lambda)}\left[I_{\infty}(K)^{s}\right]<\infty$ for $s \in[0, \kappa(\lambda)]$. 
Acknowledgements Both authors acknowledge support from the Royal Society and SP also acknowledge support from CONACyT-MEXICO Grant 351643.

Open Access This article is distributed under the terms of the Creative Commons Attribution 4.0 International License (http://creativecommons.org/licenses/by/4.0/), which permits unrestricted use, distribution, and reproduction in any medium, provided you give appropriate credit to the original author(s) and the source, provide a link to the Creative Commons license, and indicate if changes were made.

\section{Appendix}

The following result shows the a.s. uniqueness of (12) and it is needed for the proof of Proposition 1.

Lemma 2 Suppose that $\int_{[1, \infty)} x \mu(\mathrm{d} x)<\infty$ and let $K=\left(K_{t}, t \geq 0\right)$ be a Lévy process. Then for every $\lambda \geq 0, v_{t}: s \in[0, t] \mapsto v_{t}(s, \lambda, K)$ is the a.s. unique solution of the backward differential equation,

$$
\frac{\partial}{\partial s} v_{t}(s, \lambda, K)=e^{K_{s}} \psi_{0}\left(v_{t}(s, \lambda, K) e^{-K_{s}}\right), \quad v_{t}(t, \lambda, K)=\lambda,
$$

where

$$
\psi_{0}(\theta)=\psi(\theta)-\theta \psi^{\prime}(0)=\gamma^{2} \theta^{2}+\int_{(0, \infty)}\left(e^{-\theta x}-1+\theta x\right) \mu(\mathrm{d} x), \quad \theta \geq 0
$$

Proof Our proof will use a convergence argument for Lévy processes. Let $K$ be a Lévy process with characteristic $(\alpha, \sigma, \pi)$ where $\alpha \in \mathbb{R}$ is the drift term, $\sigma \geq 0$ is the Gaussian part and $\pi$ is the so-called Lévy measure satisfying $\int_{\mathbb{R} \backslash\{0\}}\left(1 \wedge z^{2}\right) \pi(\bar{d} z)<\infty$. From the Lévy-Itô decomposition (see for instance [23]), the process $K$ can be decomposed as the sum of three independent processes: $X^{(1)}$ a Brownian motion with drift, $X^{(2)}$ a compound Poisson process and $X^{(3)}$ a square-integrable martingale with an a.s. countable number of jumps on each finite time interval with magnitude less than 1 . Let $B_{\epsilon}=(-1,-\epsilon) \cup(-\epsilon, 1)$ and $M$ be a Poison random measure with characteristic measure $\mathrm{d} t \pi(\mathrm{d} x)$. Observe that the process

$$
X_{t}^{(3, \epsilon)}=\int_{[0, t]} \int_{B_{\epsilon}} x M(\mathrm{~d} s, \mathrm{~d} x)-t \int_{B_{\epsilon}} x \pi(\mathrm{d} x), \quad t \geq 0
$$

is a martingale. According to Theorem 2.10 in [23], for a fixed $t \geq 0$, there exists a deterministic subsequence $\left(\epsilon_{n}\right)_{n \in \mathbb{N}}$ such that $\left(X_{s}^{3, \epsilon_{n}}, 0 \leq s \leq t\right)$ converges uniformly to $\left(X_{s}^{3}, 0 \leq s \leq t\right)$ with probability one. We now define

$$
K_{s}^{(n)}=X_{s}^{(1)}+X_{s}^{(2)}+X_{s}^{\left(3, \epsilon_{n}\right)}, \quad s \leq t .
$$

In the sequel, we work on the space $\widetilde{\Omega}$ such that $K^{(n)}$ converges uniformly to $K$ on $[0, t]$. Note that $\psi_{0}$ is locally Lipschitz and $K^{(n)}$ is a piecewise continuous function with a finite number of discontinuities. Hence, from the Cauchy-Lipschitz Theorem, we can define a unique solution $v_{t}^{n}\left(\cdot, \lambda, K^{(n)}\right)$ of the backward differential equation:

$$
\frac{\partial}{\partial s} v_{t}^{n}\left(s, \lambda, K^{(n)}\right)=e^{K_{s}^{(n)}} \psi_{0}\left(v_{t}^{n}\left(s, \lambda, K^{(n)}\right) e^{-K_{s}^{(n)}}\right), \quad v_{t}^{n}\left(t, \lambda, K^{(n)}\right)=\lambda .
$$


In order to prove our result, we show that the sequence $\left(v^{n}(s):=v_{t}^{n}\left(s, \lambda, K^{(n)}\right), s \leq t\right)_{n \in \mathbb{N}}$ converges to a unique solution of (24) on $\widetilde{\Omega}$. With this purpose in mind, we define

$$
S=\sup _{s \in[0, t], n \in \mathbb{N}}\left\{e^{K_{s}^{(n)}}, e^{-K_{s}^{(n)}}, e^{K_{s}}, e^{-K_{s}}\right\},
$$

which turns out to be finite from the uniform convergence of $K^{(n)}$ to $K$. Since $\psi_{0} \geq 0$, we have that $v^{n}$ is increasing and $v^{n}(s) \leq \lambda$ for $s \leq t$ and $n \in \mathbb{N}$. On the other hand, since $\psi_{0}$ is a convex and increasing, we deduce that for any $0 \leq \zeta \leq \eta \leq \lambda S$, the following inequality holds

$$
0 \leq \frac{\psi_{0}(\eta)-\psi_{0}(\zeta)}{\eta-\zeta} \leq \psi_{0}^{\prime}(\eta) \leq \psi_{0}^{\prime}(\lambda S)=: C
$$

For simplicity, we denote for all $v \geq 0$,

$$
\psi^{n}(s, v)=e^{K_{s}^{(n)}} \psi_{0}\left(v e^{-K_{s}^{(n)}}\right) \quad \text { and } \quad \psi^{\infty}(s, v)=e^{K_{s}} \psi_{0}\left(v e^{-K_{s}}\right) .
$$

We then observe that for any $0 \leq s \leq t$ and $n, m \in \mathbb{N}$,

$$
\left|v^{n}(s)-v^{m}(s)\right| \leq \int_{s}^{t}\left(R^{n}(u)+R^{m}(u)\right) \mathrm{d} u+\int_{s}^{t}\left|\psi^{\infty}\left(u, v^{n}(u)\right)-\psi^{\infty}\left(u, v^{m}(u)\right)\right| \mathrm{d} u,
$$

where for any $u \in[0, t]$,

$$
\begin{aligned}
R^{n}(u) & :=\left|\psi^{n}\left(u, v^{n}(u)\right)-\psi^{\infty}\left(u, v^{n}(u)\right)\right| \\
& \leq e^{K_{u}^{(n)}}\left|\psi_{0}\left(v^{n}(u) e^{-K_{u}^{(n)}}\right)-\psi_{0}\left(v^{n}(u) e^{-K_{u}}\right)\right|+\psi_{0}\left(v^{n}(u) e^{-K_{u}}\right)\left|e^{K_{u}^{(n)}}-e^{K_{u}}\right| .
\end{aligned}
$$

Next, using (25) and (26), we deduce

$$
\begin{aligned}
R^{n}(u) & \leq S C \lambda\left|e^{-K_{u}^{(n)}}-e^{-K_{u}}\right|+\psi_{0}(S \lambda)\left|e^{K_{u}^{(n)}}-e^{K_{u}}\right| \\
& \leq\left(S C \lambda+S \psi_{0}(S \lambda)\right) \sup _{u \in[0, t]}\left\{\left|e^{K_{u}^{(n)}}-e^{K_{u}}\right|,\left|e^{-K_{u}^{(n)}}-e^{-K_{u}}\right|\right\}=: s_{n} .
\end{aligned}
$$

From similar arguments, we obtain

$$
\left|\psi^{\infty}\left(u, v^{n}(u)\right)-\psi^{\infty}\left(u, v^{m}(u)\right)\right| \leq C\left|v^{n}(u)-v^{m}(u)\right| .
$$

Therefore,

$$
\left|v^{n}(s)-v^{m}(s)\right| \leq R_{n, m}(s)+C \int_{s}^{t}\left|v^{n}(u)-v^{m}(u)\right| \mathrm{d} u,
$$

where

$$
R_{n, m}(s)=\int_{s}^{t}\left(R^{n}(u)+R^{m}(u)\right) \mathrm{d} u .
$$

Gronwall's lemma yields that for all $0 \leq s \leq t$,

$$
\left|v^{n}(s)-v^{m}(s)\right| \leq R_{n, m}(s)+C \int_{s}^{t} R_{n, m}(u) e^{C(u-s)} \mathrm{d} u .
$$


Now, recalling that $R^{n}(u) \leq s_{n}$ and $R_{n, m}(u) \leq\left(s_{n}+s_{m}\right) t$, we get that for every $N \in \mathbb{N}$,

$$
\sup _{n, m \geq N, s \in[0, t]}\left|v^{n}(s)-v^{m}(s)\right| \leq t e^{t} \sup _{n, m \geq N}\left(s_{n}+s_{m}\right) .
$$

Moreover since $s_{n} \rightarrow 0$, we deduce that $\left(v^{n}(s), s \leq t\right)_{n \in \mathbb{N}}$ is a Cauchy sequence under the uniform norm on $\widetilde{\Omega}$. In other words, for any $\omega \in \widetilde{\Omega}$ there exists a continuous function $v^{*}$ on $[0, t]$ such that $v^{n} \rightarrow v^{*}$ as $n$ goes to $\infty$. We define the function $v: \Omega \times[0, t] \rightarrow[0, \infty]$ as follows

$$
v(s)= \begin{cases}v^{*}(s) & \text { if } \omega \in \widetilde{\Omega}, \\ 0 & \text { elsewhere }\end{cases}
$$

Let $s \in[0, t]$ and $n \in \mathbb{N}$, then

$$
\begin{aligned}
\left|v(s)-\int_{s}^{t} \psi^{\infty}(s, v(s)) \mathrm{d} s-\lambda\right| \leq & \left|v(s)-v^{n}(s)\right|+\int_{s}^{t}\left|\psi^{n}(s, v(s))-\psi^{n}\left(s, v^{n}(s)\right)\right| \mathrm{d} s \\
& +\int_{s}^{t}\left|\psi^{\infty}(s, v(s))-\psi^{n}(s, v(s))\right| \mathrm{d} s \\
\leq & (1+C t) \sup _{s \in[0, t]}\left\{\left|v(s)-v^{n}(s)\right|\right\}+t s_{n} .
\end{aligned}
$$

By letting $n \rightarrow \infty$, we obtain our claim. The uniqueness of the solution of (24) follows from Gronwall's lemma.

\section{References}

1. Afanasyev, V.I., Böinghoff, C., Kersting, G., Vatutin, V.A.: Limit theorems for weakly subcritical branching processes in random environment. J. Theor. Probab. 25, 703-732 (2012)

2. Arista, J., Rivero, V.: Implicit renewal theory for exponential functionals of Lévy processes. Preprint (2015). arXiv: 1510.01809

3. Bansaye, V., Pardo, J.C., Smadi, C.: On the extinction of continuous state branching processes with catastrophes. Electron. J. Probab. 18(106), 1-31 (2013)

4. Bansaye, V., Simatos, F.: On the scaling limits of Galton Watson processes in varying environment. Electron. J. Probab. 20(75), 1-36 (2015)

5. Bansaye, V., Tran, V.C.: Branching Feller diffusion for cell division with parasite infection. ALEA Lat. Am. J. Probab. Math. Stat. 8, 95-127 (2011)

6. Berestycki, J., Fittipaldi, M.C., Fontbona, J.: Ray-Knight representation of flows of branching processes with competition by pruning of Lévy trees. Preprint (2015). arXiv:1506.00046

7. Bertoin, J., Le Gall, J-F.: Stochastic flows associated to coalescent processes. III. Limit theorems. Ill. J. Math. 50(1-4), 147-181 (2006)

8. Bertoin, J., Yor, M.: The entrance laws of self-similar Markov processes and exponential functionals of Lévy processes. Potential Anal. 17(4), 389-400 (2002)

9. Bertoin, J., Yor, M.: Exponential functionals of Lévy processes. Probab. Surv. 2, 191-212 (2005)

10. Böinghoff, C., Dyakonova, E.E., Kersting, G., Vatutin, V.A.: Branching processes in random environment which extinct at a given moment. Markov Process. Relat. Fields 16, 329-350 (2010)

11. Böinghoff, C., Hutzenthaler, M.: Branching diffusions in random environment. Markov Process. Relat. Fields 18, 269-310 (2012)

12. Caballero, M.E., Lambert, A., Uribe Bravo, G.: Proof(s) of the Lamperti representation of continuousstate branching processes. Probab. Surv. 6, 62-89 (2009)

13. Dawson, D.A., Li, Z.: Skew convolution semigroups and affine Markov processes. Ann. Probab. 34(3), 1103-1142 (2006)

14. Dawson, D.A., Li, Z.: Stochastic equations, flows and measure-valued processes. Ann. Probab. 40, 813857 (2012) 
15. Doney, R., Maller, R.: Stability and attraction to normality for Lévy processes at zero and at infinity. J. Theor. Probab. 15, 751-792 (2002)

16. Erickson, K., Maller, R.: Generalised Ornstein-Uhlenbeck processes and the convergence of Lévy integrals. In: Émery, M., Ledoux, M., Yor, M. (eds.) Séminaire de Probabilités XXXVIII, pp. $70-94$ (2005)

17. Evans, S.E., Hening, A., Schreiber, S.: Protected polymorphisms and evolutionary stability of patchselection strategies in stochastic environments. J. Math. Biol. 71, 325-359 (2015)

18. Fu, Z., Li, Z.: Stochastic equations of non-negative processes with jumps. Stoch. Process. Appl. 120, 306-330 (2010)

19. Grimvall, A.: On the convergence of sequences of branching processes. Ann. Probab. 2, 1027-1045 (1974)

20. He, H., Li, Z., Xu, W.: Continuous-state branching processes in Levy random environments. Preprint (2016). arXiv: 1601.04808

21. Ikeda, N., Watanabe, S.: Stochastic Differential Equations and Diffusion Processes, 2nd edn. NorthHolland, Amsterdam (1989)

22. Kawazu, K., Watanabe, S.: Branching processes with immigration and related limit theorems. Theory Probab. Appl. 16, 36-54 (1971)

23. Kyprianou, A.: Fluctuations of Lévy Processes with Applications: Introductory Lectures, 2nd. edn. Springer, Berlin (2014)

24. Keiding, N.: Extinction and exponential growth in random environments. Theor. Popul. Biol. 8, 49-63 (1975)

25. Kurtz, T.: Diffusion approximations for branching processes. In: Branching Processes (Conf. Saint Hippolyte, Que.) Adv. Probab. Related Topics, vol. 5, pp. 269-292. Dekker, New York (1976)

26. Lambert, A.: The branching process with logistic growth. Ann. Appl. Probab. 15, 1506-1535 (2005)

27. Li, Y., Liu, Q., Gao, Z., Wang, H.: Asymptotic properties of supercritical branching processes in random environments. Front. Math. China 9(4), 1673-3452 (2014)

28. Li, Z., Xu, W.: Asymptotic results for exponential functionals of Lévy processes. Preprint (2016). arXiv: 1601.02363

29. Li, Z., Pu, F.: Strong solutions of jump-type stochastic equations. Electron. Commun. Probab. 17(33), 1-13 (2012)

30. Ma, R.: Lamperti transformation for continuous-state branching processes with competition and applications. Stat. Probab. Lett. 107, 11-17 (2015)

31. Maulik, K., Zwart, B.: Tail asymptotics for exponential functionals of Lévy processes. Stoch. Process. Appl. 116, 156-177 (2006)

32. Palau, S., Pardo, J.C.: Continuous state branching processes in random environment: the Brownian case. Stoch. Process. Appl. 127(3), 957-994 (2017)

33. Palau, S., Pardo, J.C., Smadi, C.: Asymptotic behaviour of exponential functionals of Lévy processes with applications to random processes in random environment. ALEA Lat. Am. J. Probab. Math. Stat. 13(2), 1235-1258 (2016)

34. Pardoux, E.: Probabilistic Models of Population Evolution. Mathematical Biosciences Institute Lecture Series. Stochastics in Biological Systems. Springer, Berlin (2016)

35. Protter, P.: Stochastic Integration and Differential Equations. Stochastic Modelling and Applied Probability. Springer, Berlin (2005)

36. Sato, K.: Lévy Processes and Infinitely Divisible Distributions. Cambridge University Press, Cambridge (1999)

37. Smith, W.L., Wilkinson, W.E.: On branching processes in random environments. Ann. Math. Stat. 40, 814-827 (1969) 